\title{
Comments: "Preoperative anxiety induces chronic postoperative pain by activating astrocytes in the anterior cingulate cortex region"
}

\author{
Baohong Xue \\ (iD) Lianping $\mathrm{He}^{1,2}$ \\ 1. College of experience industry, Anhui polytechnic university, Wuhu, Anhui 241000 , China \\ 2. Department of Immunology, Nanjing medical university, Nanjing, Jiangsu 211166, China

Dear Editor,

It was with great interest that we read the study by Daming $\mathrm{Gu}^{1}$ and colleagues in which they demonstrated that anxiety can induce chronic pain by activating astrocytes in the anterior cingulate cortex region. The authors believe that the activation of astrocytes can induce chronic pain. In our opinion, more experiments are necessary to reach any conclusion.

To begin with, the authors should confirm the success of the mouse model of anxiety since no related experiment was performed to evaluate the anxiety model prior to the operation. In order to examine the anxiety model, the authors could use the elevated plus maze and open field tests ${ }^{2}$. Additionally, the operation skill also has great influence on chronic pain, and the authors should address this issue.

There are many risk factors for chronic pain, such as inflammation, which plays an important role in regulating this type of pain. Thus, we wonder why the authors only focused on the activation of astrocytes. In conclusion, more experiments should be done to exclude the role of corticotropin-releasing factors, inflammation factors, and noradrenalin ${ }^{3}$.

\section{Acknowledgment}

This work was supported by the scientific research innovation program of graduate students of the Jiangsu province (grant number KYCX17_1267)

\section{REFERENCES}

1. Damin Gu, Minmin Zhou, Chao Han, Daoyun Lei, Songhui Xie, Yanbo Yuan, Tieliang Ma - Preoperative anxiety induces chronic postoperative pain by activating astrocytes in anterior cingulate cortex region. Rev Assoc Med Bras 2019; 65(9)1174-1180

2. Wei, D. Zhao, N. Xie, L. Huang, B. Zhuang, Z. Tang, Y., et al. Electroacupuncture and Moxibustion Improved Anxiety Behavior in DSS-Induced Colitis Mice. Gastroenterol Res Pract 2019;2019: 2345890.

3. Phyomaung, P. P. Dubowitz, J. Cicuttini, F. M. Fernando, S. Wluka, A. E. Raaijmaakers, P., et al. Are depression, anxiety and poor mental health risk factors for knee pain? A systematic review. BMC Musculoskelet Disord 2014;15: 10. 